\title{
Tooth Crack Severity Assessment in the Early Stage of Crack Propagation Using Gearbox Dynamic Model
}

\author{
Xingkai Yang ${ }^{1}$, Ming J. Zuo ${ }^{2}$, and Zhigang Tian ${ }^{3}$ \\ 1,2,3 Department of Mechanical Engineering, Edmonton, Alberta, T6G 2G8, Canada \\ xingkai2@ualberta.ca \\ ming.zuo @ualberta.ca \\ ztian@ualberta.ca
}

\begin{abstract}
Localized tooth crack in gearboxes may be reflected in impulse components of gearbox vibration signals. Crack induced impulses have been used for crack detection and fault diagnosis. In reported studies, researchers have used statistical indicators of the identified impulses, such as root mean square $(R M S)$ and kurtosis, to track the growth of crack. These reported statistical indicators are only effective when crack levels are high and they are unable to detect tooth crack and assess crack severity in the early stage of crack propagation. In addition, no reported studies have focused on studying how tooth crack level affects crack induced impulses. Specifically, what the dominant segments of crack induced impulses are and which segment is affected most by crack growth within a certain crack level range. This paper uses dynamic modeling to study how crack level affects crack induced impulses. First, impulses are generated with a spur gearbox dynamic model under constant working conditions. Second, an exponentially damped sinusoidal model is utilized to fit the impulses and the Matrix Pencil Method is used for model parameter estimation. Finally, relationships between crack level and impulses are studied based on the obtained model parameters. The results have shown that the segments in the fifth and the sixth frequency bands of impulses are two dominant segments, while other segments have little contribution, for the gearbox system under investigation. Within a certain crack level range, there exists an impulse segment which is most affected by the crack level. In terms of the early stage of crack propagation, the segment in the sixth frequency band of the impulse is most affected by crack growth. On this basis, three new statistical indicators have been developed with the segment in sixth frequency band of the impulse and have shown their effectiveness for tooth crack severity level assessment in the early stage of crack
\end{abstract}

Xingkai Yang et al. This is an open-access article distributed under the terms of the Creative Commons Attribution 3.0 United States License, which permits unrestricted use, distribution, and reproduction in any medium, provided the original author and source are credited. propagation. These results have good potential for detection and severity assessment of early tooth cracks in gearboxes.

\section{INTRODUCTION}

Gearboxes are widely used in industrial applications, such as wind turbines and railway vehicles. Due to harsh working environments, gearboxes are easily suffering from various faults, such as tooth crack, tooth breakage, pitting and spalling. Once faults occur, it may cause great damage to the whole system, even leading to catastrophic accidents. Tooth crack accounts for a large proportion of gearbox faults. Therefore, research on tooth crack detection and diagnosis has attracted lots of attention during the past decades, especially in the early stage of tooth crack propagation.

For healthy gearboxes, their vibration signals are mainly composed of the fundamental gear meshing frequency and its harmonics (Randall, 1982). But when there is a localized gear tooth crack, vibration signals will be affected since impulses with short time duration are introduced. It's believed that the short duration impulse is induced by the localized tooth crack (Wang, 2001). Crack induced impulses in vibration signals of faulty gearboxes could be extracted and used to detect the existence of tooth crack and assess crack severity (Wang \& Wong, 2002), (Tian, Zuo, \& Wu, 2012).

There are two groups of vibration-based methods for tooth crack detection and diagnosis. The first group is based on gearbox vibration data collected from experimental tests or field applications while the second one relies on the simulated vibration responses obtained with gearbox dynamic models. Compared with real vibration data based methods, simulated vibration response based methods have two advantages (Tian et al., 2012): (1) There is no environmental noise in gearbox vibration responses, which helps researchers to only focus on analyzing crack induced impulses and develop effective tooth crack diagnosis methods; (2) It's easy to simulate various tooth crack severity levels with well-developed dynamic models, which is helpful to study relationships between crack level and vibration signals. Therefore, methods based on 
simulated vibration responses become more and more popular for tooth crack diagnosis.

For simulated vibration response based methods, vibration responses obtained with gearbox dynamic models are processed to conduct tooth crack detection and diagnosis. A new residual signal generation method by subtracting the entire vibration response of healthy gear from that of the faulty gear was presented in (Wu, Zuo, \& Parey, 2008). The obtained residual signals can be seen as crack induced impulses in this situation. The root mean square of the impulse $(R M S I)$ and the kurtosis of the impulse (KURI) became more sensitive to crack growth than those obtained with the original vibration response. But RMSI and KURI was ineffective for early crack detection since their increase was pretty small in the early stage of crack propagation, i.e., they are insensitive to early crack level. A new indicator, root mean square of the segment of crack induced impulse in the first three tooth meshing cycles (RMSI3), was developed (Tian et al., 2012). They showed that RMSI3 was more sensitive for reflecting crack growth than RMSI and KURI. But the increasing rate of $R M S I 3$ still remains relatively low in the early crack stage, which means it is not effective for assessing tooth crack level. Recently, a method for gear tooth crack diagnosis based on modelling the crack induced impulse with Laplace wavelet and maximum correlation coefficient has been reported (Wang \& Shao, 2017). They tried to describe the mode of crack induced impulse with a parametric-Laplace wavelet technique and determine the most suitable wavelet base function with the maximum correlation coefficient criterion. The correlation coefficient between the optimal Laplace wavelet base function and the impulse was designed as a health condition indicator for crack diagnosis. The results showed that the indicator increases as crack propagates. But there are some drawbacks: (1) it assumed that there exists only one mode in the impulse, which may be improper since it may contain several modes; (2) the developed indicator has very slight increase in the early stage of crack propagation and cannot show obvious increase even in large crack levels; (3) no attempt was made to analyze how crack induced impulses change as tooth crack gets severer. Therefore, further studies should be conducted to investigate how crack level affects crack induced impulses. If the dominant impulse segments and the segment most affected by crack level in the early crack stage could be known, then statistical indicators developed with this specific impulse segment are expected to have good performance for crack severity assessment in the early stage of crack propagation.

In this paper, based on a dynamic model of one-stage fixedaxis spur gearbox with local tooth crack under constant working condition, crack induced impulses are generated. They are modelled with exponentially damped sinusoids and model parameters are estimated with the Matrix Pencil Method. By processing model parameter estimates, the most dominant impulse segment and the segment more affected by crack level in certain crack level ranges are studied. Three new indicators are developed for tooth crack severity assessment in the early stage of crack propagation.

The remainder of this paper is organized as follows. In Section 2, the overall method for analyzing the crack induced impulse is illustrated with a developed procedure. The generation of crack induced impulses of various crack levels is shown in Section 3. Section 4 introduces the exponentially damped sinusoidal model and the Matrix Pencil Method. Section 5 presents the results and discussions. Finally, conclusions are drawn in Section 6.

\section{OVERAll Method}

The overall method shown in Figure 1 is developed to find out the most dominant impulse segment and the segment more affected by crack level in early crack stage. And then the specific segment is adopted to develop new health indicators for early tooth crack severity level assessment.

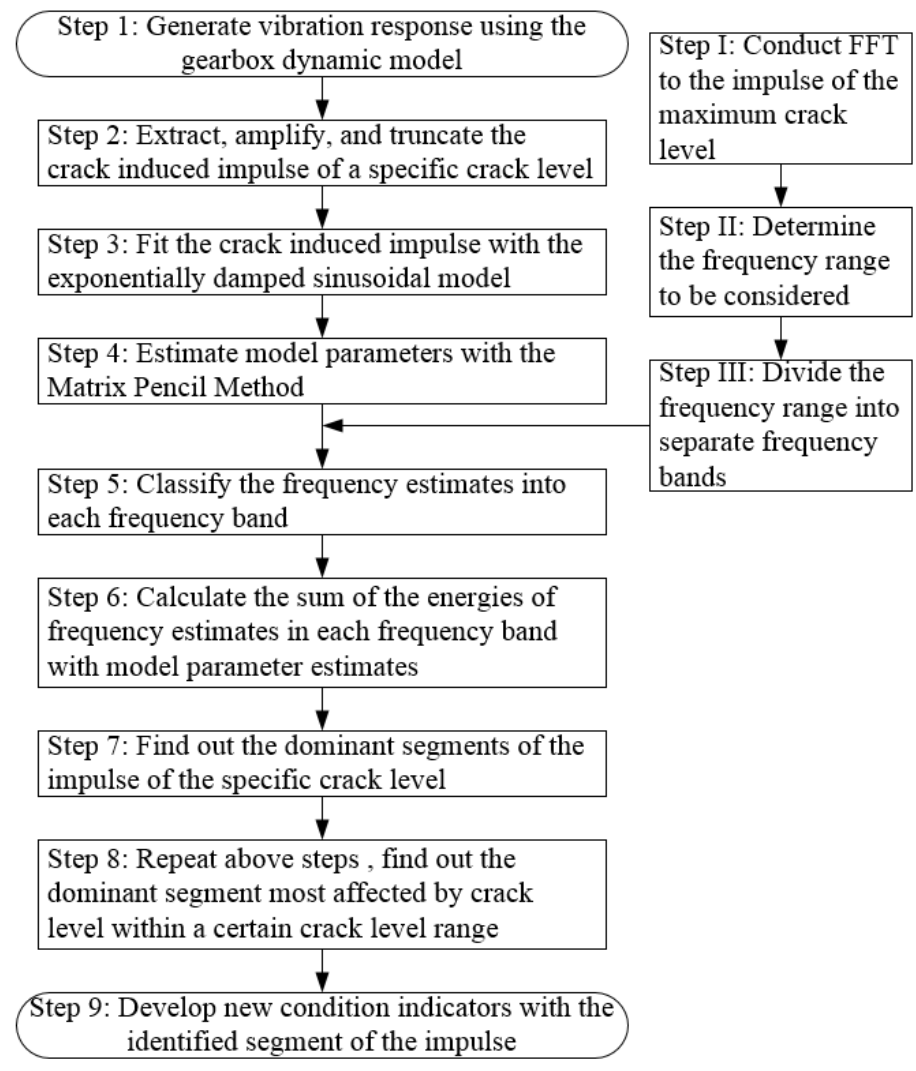

Figure 1. The procedure of the overall method.

From Figure 1, it's seen that there are nine main steps and three additional steps. These 12 steps could be divided in to several separate parts listed below.

(1) Crack induced impulse generation. This part contains Step 1 and Step 2. Crack induced impulses of all the considered crack levels are obtained; 
(2) Crack induced impulse fitting. Step 3 and Step 4 are included. For crack induced impulse of a specific crack level, it's fitted with exponentially damped sinusoidal model and model parameters are estimated with Matrix Pencil Method;

(3) Frequency range division. The Steps I, II, and III are placed in this part. Here, the frequency range to be considered and division of frequency range are determined;

(4) Post-processing of model parameter estimates. This part includes Steps 5, 6, and 7. The post-processing of model parameter estimates are conducted;

(5) The most dominant segment in a certain crack level range. This part contains Step 8. Repeat steps from Step 2 to Step 7, the most dominant segment and the segment more affected by crack level are expected to be known;

(6) Development of new health condition indicators. This part includes Step 9. New indicators are developed for early crack severity level assessment.

\section{THE GENERATION OF CRACK INDUCED IMPULSE}

In this section, how to generate the crack induced impulse with gearbox dynamic model is summarized.

\subsection{The Cracked Gear Tooth Model}

A gear tooth crack usually initiates at the tooth root fillet. Tooth crack will propagate in certain paths. In this paper, crack propagation path has been simplified as a straight line starting from tooth root fillet. The simplified cracked tooth model with maximum crack depth is shown in Figure 2.

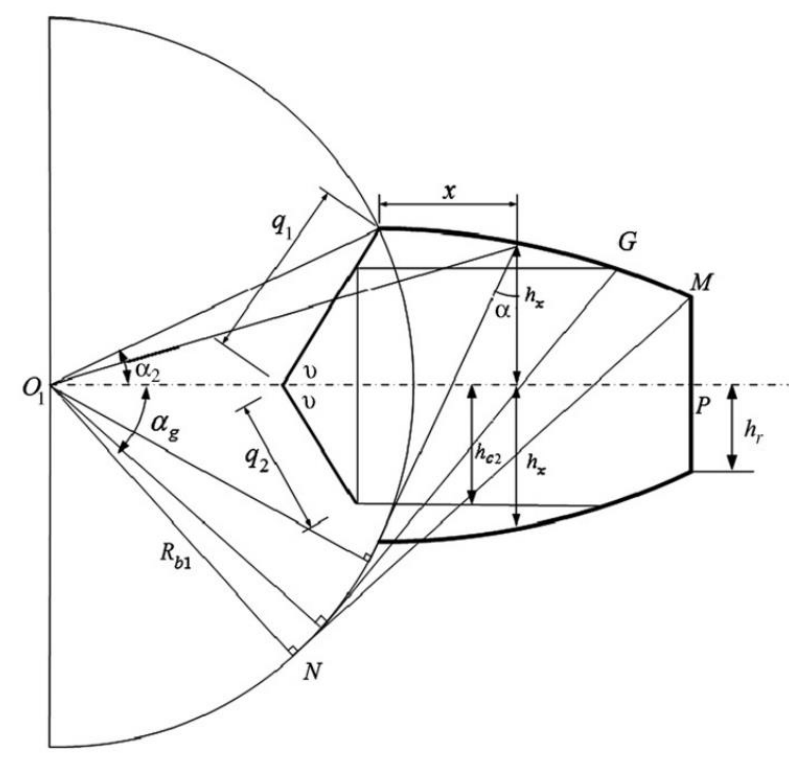

Figure 2. The cracked tooth model (Tian et al., 2012).

In Figure 2, $q_{1}$ is the maximum crack depth from the initial point of crack propagation to the central line of gear tooth, $q_{2}$ is the actual maximum crack depth in the symmetric direction. For the gearbox dynamic model in (Tian et al., 2012), $q_{1}=3.9 \mathrm{~mm}$ and $q_{2}=2.34 \mathrm{~mm}$. The percentage of a crack depth to the theoretical maximum depth is defined as the crack level $(c l)$, which is calculated with Eq. (1).

$$
c l=q /\left(2 \times q_{1}\right) \times 100 \%
$$

where $q$ is the considered crack depth varying from $0 \mathrm{~mm}$ to $6.24 \mathrm{~mm}$ with the increment of $\Delta q=0.1 \mathrm{~mm}$.

Based on Eq. (1), the crack level corresponds to an arbitrary crack depth could be calculated. When $q=0 \mathrm{~mm}$, it's the healthy gear case, $c l=0 \%$. For the minimum crack depth $q=0.1 \mathrm{~mm}, c l=1.29 \%$. When $q=6.24 \mathrm{~mm}$, i.e., actual maximum crack depth, $\mathrm{cl}=80 \%$. In this paper, the crack level range $(C L R)$ to be considered is $C L R=[1.29 \%, 80 \%]$.

\subsection{The Gearbox Dynamic Model}

The one-stage fixed-axis spur gearbox dynamic model is shown in Figure 3, which is adopted from (Tian et al., 2012).

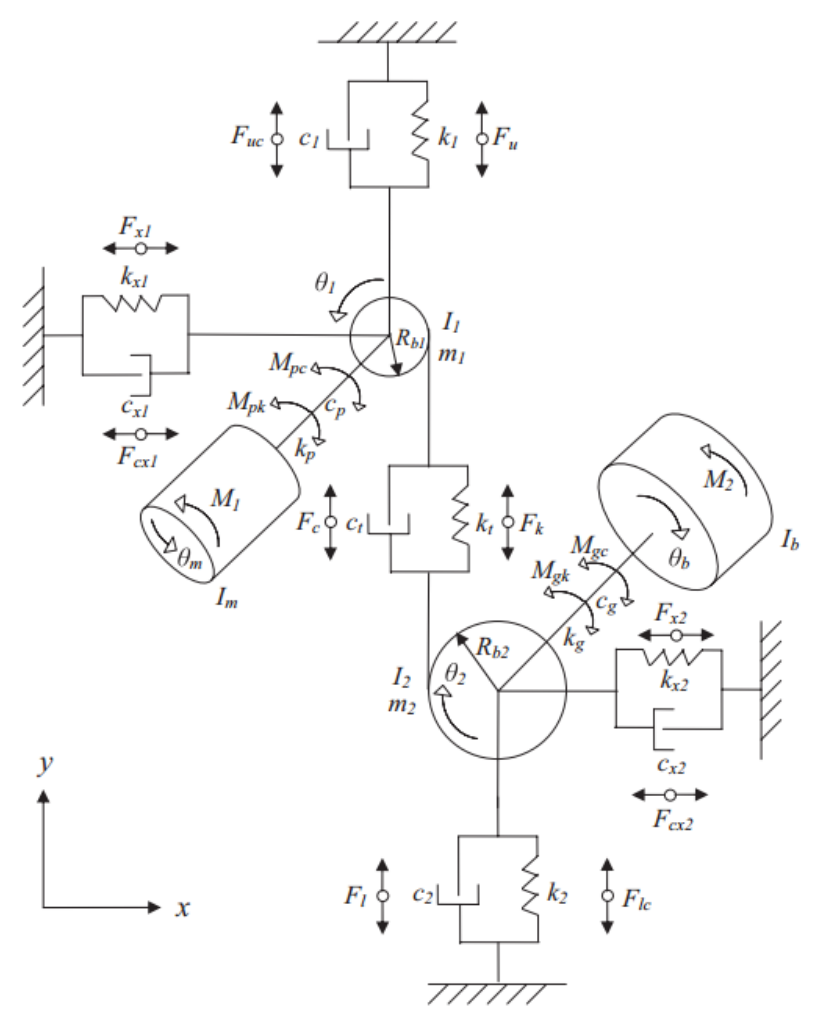

Figure 3. The gearbox dynamic model (Tian et al., 2012).

This model represents a gearbox system with eight degrees of freedom. The cracked tooth is with the pinion $m_{1}$ in Figure 3 , and its motion in y direction is studied. The number of pinion teeth is $N_{p}=19$, pinion shaft rotating frequency is $f_{s}=30 \mathrm{~Hz}$. The gear mesh frequency is $f_{\text {mesh }}=N_{p} \times f_{s}=$ $570 \mathrm{~Hz}$ and pinion rotating period is $T_{p}=1 / f_{s}=0.0333 \mathrm{~s}$. 


\subsection{The Crack Induced Impulse}

Based on the gearbox dynamic model, vibration responses of all crack levels are obtained. Then crack induced impulses are extracted using the method in (Wu et al., 2008). For example, the impulse of $80 \%$ crack level is obtained by subtracting the vibration response of the healthy gear $(\mathrm{cl}=0 \%)$ from that of the faulty gear $(\mathrm{cl}=80 \%)$. It is periodic and its amplitude is extremely small, and thus its amplitude is amplified by multiplying a factor $10^{8}$. The time-domain waveform and frequency spectrum of the amplified impulse are shown in Figure 4. Besides, it's truncated to have only one period $\left(T_{p}\right)$ to reduce computational complexity. The obtained impulse in one period is shown in Figure 5.
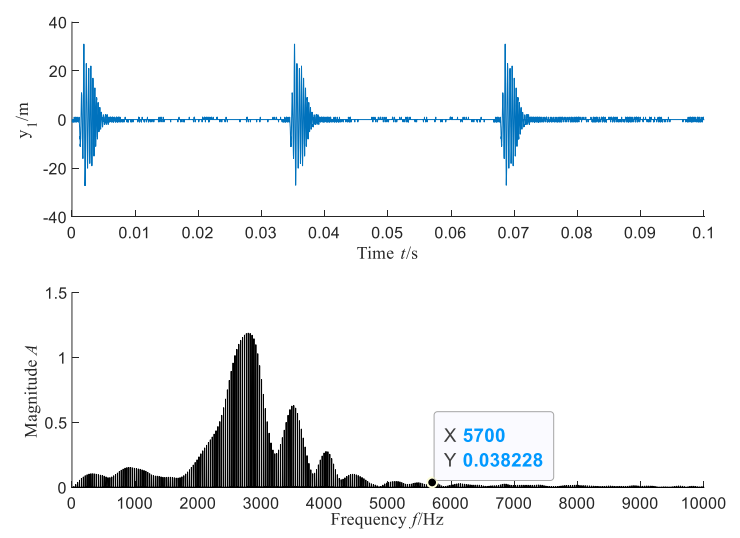

Figure 4. Time-domain waveform and spectrum of the impulse with $c l=80 \%$.

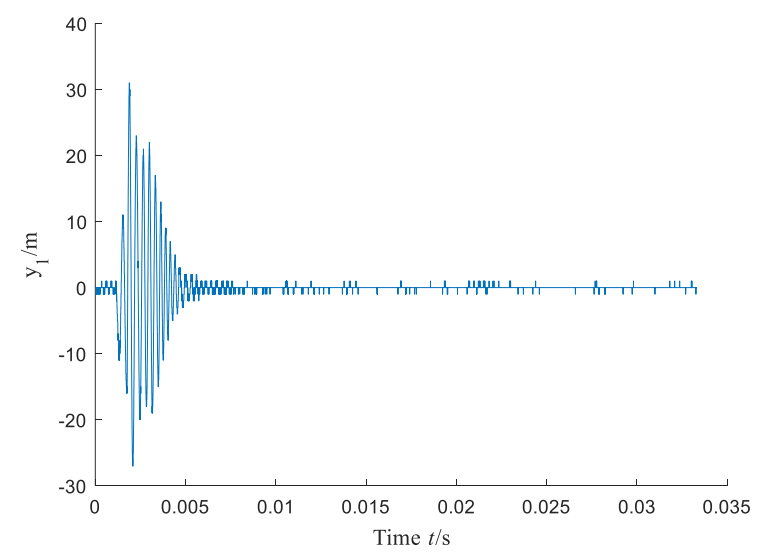

Figure 5. The impulse waveform in one period $(c l=80 \%)$.

From Figure 4, it's obvious that there are several resonant peaks in the frequency spectrum of the crack induced impulse. These resonant peaks are excited by the localized tooth crack and each resonant peak corresponds to a specific natural frequency of the gearbox system. Similar resonant peaks in the frequency spectrum of the crack induced impulse could also be found in the reported study (Li et al., 2016). But in terms of the experimental or filed vibration data of cracked gearboxes, due to the strong environmental noise and the effects of vibration transfer path and sensor bracket, it's hard to observe dominant and obvious resonant peaks in the frequency spectrums.

From Figure 4, it's also seen that spikes with $f>5700 \mathrm{~Hz}$ have very small magnitudes, which means spikes beyond $5700 \mathrm{~Hz}$ have little contribution to the impulses and can be negligible. It can hold for impulses of all the other crack levels. So the frequency range to be considered is $[0 \mathrm{~Hz}, 5700 \mathrm{~Hz}]$. It is divided into several frequency bands (FBs). The upper bound, center, and lower bound of the $i^{\text {th }}$ $\mathrm{FB}$, i.e. $F B_{i}$, are $(i+0.5) \times f_{\text {mesh }}, i \times f_{\text {mesh }}$, and $(i-$ $0.5) \times f_{\text {mesh }}$, respectively. Here $i$ is the number of FB and $i=0,1, \ldots, 10$. The spread of each FB equals $f_{\text {mesh }}$, except the first one and the last one since they only have a spread of half $f_{\text {mesh }}$. The division results are tabulated in in Table 1.

Table 1. Eleven frequency bands of the frequency range.

\begin{tabular}{cccccc}
\hline $\mathrm{FB}_{0}$ & $\mathrm{FB}_{1}$ & $\mathrm{FB}_{2}$ & $\mathrm{FB}_{3}$ & $\mathrm{FB}_{4}$ & $\mathrm{FB}_{5}$ \\
\hline$[0$, & {$[285$,} & {$[855$,} & {$[1425$,} & {$[1995$,} & {$[2565$,} \\
$285]$ & $855]$ & $1425]$ & $1995]$ & $2565]$ & $3135]$ \\
\hline $\mathrm{FB}_{6}$ & $\mathrm{FB}_{7}$ & $\mathrm{FB}_{8}$ & $\mathrm{FB}_{9}$ & $\mathrm{FB}_{10}$ & \\
\hline$[3135$, & {$[3705$,} & {$[4275$,} & {$[4845$,} & {$[5415$,} & \\
$3705]$ & $4275]$ & $4845]$ & $5415]$ & $5700]$ & \\
\hline
\end{tabular}

\section{CRACK INDUCED IMPULSE MODELling}

In this section, how to model cracked induced impulses and estimate model parameters is introduced.

\subsection{Exponentially Damped Sinusoidal Model}

Some reported studies claimed that the vibration signal of a cracked spur gear has a transient nature and could be modelled using impulse responses (Antoni \& Randall, 2006). For a single degree of freedom vibration system considering the effect of damping, its impulse response could be represented with an exponentially damped sinusoid. The exponentially damped sinusoid could be seen as a modulated signal whose carrier frequency and modulation frequency are the system undamped natural frequency and the damped natural frequency, respectively. For the considered spur gearbox system in Figure 3, it's a system with multiple degrees of freedom, so it has multiple natural frequencies. When there exists a tooth crack, it will excite multiple natural frequencies (Li et al., 2016). So the impulse response could be represented using the sum of several exponentially damped sinusoids. If only a resonant frequency is considered, then the crack induced impulse could be modelled with a single exponentially damped sinusoid (Zhao \& Jia, 2017).

There is another way to model the crack induced impulse, namely to treat it as a modulated signal whose carrier and modulation parts are harmonics and a short-period rectangular pulse, respectively (Randall, 1982). But this 
model is not conformed to the solutions of the vibration equations of gearbox system with multiple degrees of freedom, and so it's not adopted to model the cracked induced impulse in this paper.

Besides, the impulse in Figure 5 has almost the same waveform pattern as speech signals (Kumaresan \& Tufts, 1982). In the research area of speech signal analysis, transient speech signals are always modelled with exponentially damped sinusoids (Boyer \& Abed-Meraim, 2004).

Therefore, it's reasonable and feasible to adopt the exponentially damped sinusoidal model in Eq. (2) to mathematically represent the crack induced impulses. In Eq. (2), the $i^{t h}$ exponentially damped sinusoid is seen as the $i^{\text {th }}$ component of the crack induced impulse.

$$
y(\mathrm{t})=\sum_{i=1}^{M} A_{i} e^{\left(D_{i} t\right)} \cos \left(2 \pi f_{i} t+\theta_{i}\right)
$$

where $M$ is the number of the exponentially damped sinusoids; $A_{i}$ is the amplitude, $D_{i}$ the damping factor, $f_{i}$ the frequency, and $\theta_{i}$ the initial phase of the $i^{t h}$ exponentially damped sinusoid, respectively.

Please note this model needs to be modified to represent the measured cracked gearbox impulse signals if the effects of vibration transfer path and sensor bracket are considered.

\subsection{The Matrix Pencil Method}

The problem of estimating model parameters is equivalent to solving the nonlinear least-squares problem in Eq. (3).

$$
\hat{p}=\arg \min _{p}\left\|y-\sum_{i=1}^{M} A_{i} e^{\left(D_{i} t\right)} \cos \left(2 \pi f_{i} t+\theta_{i}\right)\right\|^{2}
$$

where $p=\left(M, A_{1}, f_{1}, \theta_{1}, D_{1}, \ldots, A_{M}, f_{M}, \theta_{M}, D_{M}\right)$ is the unknown model parameter vector to be estimated.

To solve the nonlinear least-squares problem in Eq. (3), either nonlinear methods or linear methods could work. For nonlinear method, the Hankel Total Least Square (HTLS) method (Lemmerling \& Van Huffel, 2001) works. For linear methods, the Prony's method (Potts \& Tasche, 2010), Linear Prediction Singular Value Decomposition (LPSVD) (Tufts \& Kumaresan, 1982), and the Matrix Pencil Method (Hua \& Sarkar, 1990) had shown their effectiveness. The comparison results presented in (Liu et al., 2007) concluded that the Matrix Pencil Method is most robust to noise and computationally efficient among those methods. Therefore, the Matrix Pencil Method is employed to conduct model determination while the Prony's method, HTLS, and LPSVD are not considered.

The Matrix Pencil Method could be seen as a combination of the linear matrix operation and nonlinear optimization for estimating the unknown model parameters in Equation (3) (Hua \& Sarkar, 1990). Its linear matrix operation is used for estimating model order $\mathrm{M}$, frequencies $f_{i}$ and damping factors $D_{i}$, while the nonlinear optimization step is adopted to estimate the amplitudes $A_{i}$ and phases $\theta_{i}$. This special property of the Matrix Pencil Method makes it suitable for doing a linear model with high computational efficiency and estimation accuracy.

\subsection{The Fitting Result}

With model parameter estimates, the impulse can be reconstructed. The coefficient of determination $\left(R^{2}\right)$ defined in Eq. (4) is used to evaluate the fitting accuracy. It's a measure of how well a model can fit the data. Its value falls between 0 and 1 , the higher the value, the better the model approximation accuracy.

$$
R^{2}=1-\frac{\left\|y_{o}-y_{r}\right\|^{2}}{\left\|y_{o}\right\|^{2}}
$$

where $y_{o}$ is the original signal, and $y_{r}$ is the reconstructed signal.

Use the impulse with $c l=80 \%$ shown in Figure 5 as an example. This original impulse is named $y_{o}$. Based on model parameter estimates, the reconstructed impulse $y_{r}$ is obtained. The coefficient of determination is $R^{2}=0.994$, which means the impulse modelling can get a pretty high fitting accuracy. The original impulse and the reconstructed version are plotted in Figure 6. It's seen that the reconstructed impulse has well agreed with the original one. For impulses of other crack levels, they can also be well fitted.

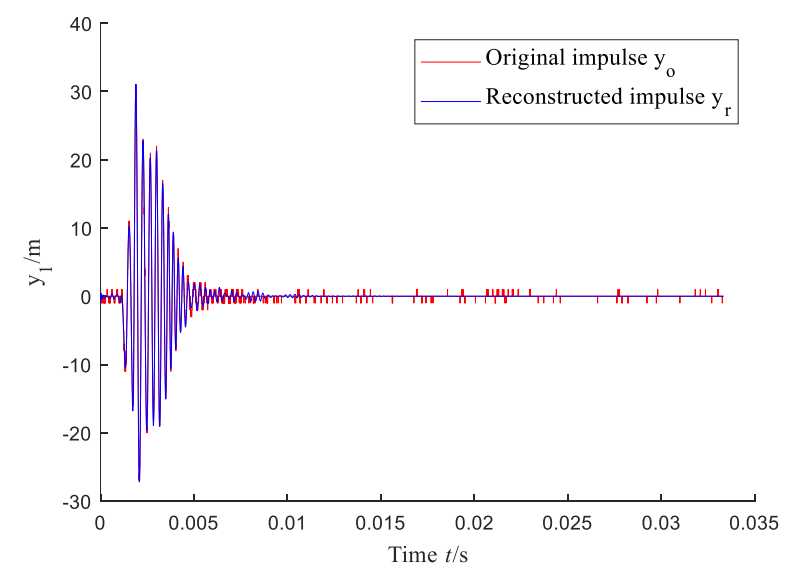

Figure 6. The original impulse and its reconstructed version with $c l=80 \%$ in one period.

\section{RESULTS AND DISCUSSIONS}

For crack induced impulses with crack levels in $C L R=$ $[1.29 \%, 80 \%]$, they can be represented with identical model structure but with different model parameters. Model parameters are closely related with crack level. Therefore, how impulses change with crack levels are studied with model parameter estimates. 


\subsection{Post-processing of Model Parameter Estimates}

For each crack level, the corresponding crack induced impulse could be decomposed into several components with the Matrix Pencil Method. Then the sum of the energies of components in $F B_{i}(i=0,1, \ldots, 10)$ is calculated and is named $E_{F B_{i}}$, and the sum of the components in $F B_{i}$ is called impulse segment $y_{F B_{i}}$. For conciseness, only energy results of impulse with $c l=58.06 \%$ are illustrated in Figure 7 .

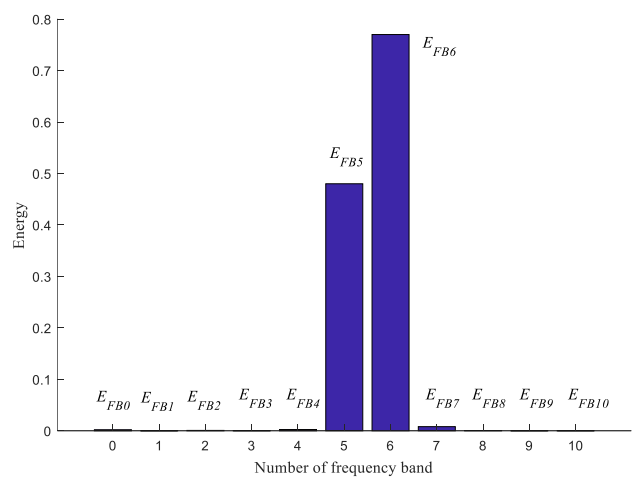

Figure 7. Energy in each FB of impulse with $c l=58.06 \%$.

To find out the dominant impulse segments, energy is used as the selection criterion. Specifically, impulse segment in a specific FB with high energy is considered to be dominant and the one with biggest energy is seen as the most dominant segment. From Figure 7, it's seen that $E_{F B_{5}}$ and $E_{F B_{6}}$ are much greater than the others. Therefore, impulse segments in $F B_{5}$ and that in $F B_{6}$ are considered as two dominant impulse segments for $\mathrm{cl}=58.06 \%$. Here, name these two segments $y_{F B_{5}}$ and $y_{F B_{6}}$, respectively.

\subsection{Two dominant segments of crack induced impulses}

Following the method in Subsection 5.1, values of $E_{F B_{i}}(i=$ $0,1, \ldots, 10)$ for impulses of all the considered crack levels are calculated and compared. It's found that the observation, namely $y_{F B_{5}}$ and $y_{F B_{6}}$ are two dominant impulse segments, can still hold for all the crack levels in CLR $=[1.29 \%$, 80\%]. But the most dominant segment can be different in different crack level ranges. How $E_{F B_{5}}, E_{F B_{6}}$, and the logarithm of their quotient, i.e., $L Q=\log _{10}\left(E_{F B_{5}} / E_{F B_{6}}\right)$, change versus the crack level are plotted in Figure 8.

From Figure 8, several useful observations are obtained and listed below.

(1) In $C L R_{1}=[1.29 \%, 23.23 \%]$ and $C L R_{2}=[23.23 \%$, $58.06 \%$ ], $L Q<0$, which means $E_{F B_{5}}<E_{F B_{6}}$. It's concluded that in these two ranges, $y_{F B_{6}}$ is the most dominant segment;

(2) In $C L R_{1}=[1.29 \%, 23.23 \%], L Q$ is much smaller which indicates the increasing rate of $E_{F B_{6}}$ is much bigger than that of $E_{F B_{5}}$, thus meaning segment $y_{F B_{6}}$ is most affected by crack level. $C L R_{1}$ is seen as the early stage of crack propagation (Tian et al., 2012). Therefore, $y_{F B_{6}}$ is most affected by crack level in the early crack stage;

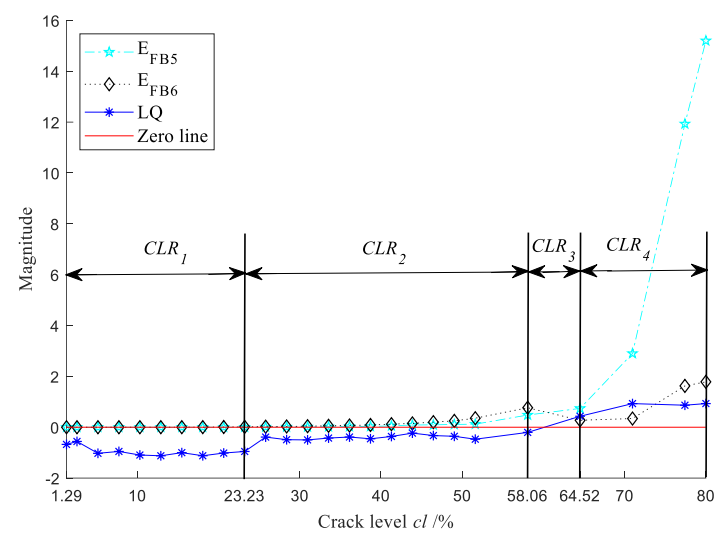

Figure 8. The values of $E_{\mathrm{FB}_{5}}, E_{\mathrm{FB}_{6}}$, and $L Q$ versus crack levels $(C L R=[1.29 \%, 80 \%])$.

(3) In $C L R_{2}=[23.23 \%, 58.06 \%]$, the value of $L Q$ is bigger than that in $C L R_{1}$, but still smaller than 0 . This means that $E_{F B_{5}}$ is still smaller than $E_{F B_{6}}$, but their increasing rates versus crack level are almost the same. This further indicates that in $C L R_{2}, y_{F B_{6}}$ is less affected by the crack level than it's in $C L R_{1}$;

(4) In $C L R_{3}=[58.06 \%, 64.52 \%], L Q$ is increasing and finally exceeds 0 , which indicates $E_{F B_{5}}$ is gradually approaching $E_{F B_{6}}$ and eventually bigger than $E_{F B_{6}}$. This means energy of the impulse is gradually transferred from segment $y_{F B_{6}}$ to $y_{F B_{5}}$. This is a phenomenon of energy transfer from one impulse segment to another;

(5) In $C L R_{4}=[64.52 \%, 80 \%], L Q>0$, which means $E_{F B_{5}}>E_{F B_{6}}$, indicating that $y_{F B_{5}}$ is the most dominant impulse segment.

\subsection{Three new statistical indicators}

Three new statistical indicators are developed with the dominant segment most affected by crack level in the early stage of crack propagation. Here, this specific impulse segment is named $y_{F B_{m}}$ and its related FB is named $F B_{m}$. The three new statistical indicators are defined as follows.

1) $S E_{F B_{m}}$ : sum of energies of the components of $y_{F B_{m}}$;

2) $S R M S_{F B_{m}}$ : sum of root mean square values of the components of $y_{F B_{m}}$;

3) $P M S_{F B_{m}}$ : peak magnitude of the spikes in $F B_{m}$.

For the reported gearbox system in (Tian et al., 2012), based on the observations in Subsection 5.2, it's found that $y_{F B_{6}}$ is the dominant impulse segment most affected by the crack level in the early stage of crack propagation. This means $y_{F B_{6}}$ 
will have biggest change as the crack level gets larger. Three new statistical indicators are developed with segment $y_{F B_{6}}$ and are shown in below three equations. It's expected that these three new indicators could have much higher increasing rates than those developed with the original impulses or the whole vibration signals.

$$
\begin{gathered}
S E_{F B_{m}}=\sum_{i=1}^{I} E_{i},(i=1,2, \ldots, I) \\
S R M S_{F B_{m}}=\sum_{i=1}^{I} R M S_{i},(i=1,2, \ldots, I) \\
P M S_{F B_{m}}=\max \left(A_{i}\right),(i=1,2, \ldots, I)
\end{gathered}
$$

where $I$ is the number of components in $F B_{6} ; E_{i}$ and $R M S_{i}$ are energy and root mean square of $i^{\text {th }}$ component of $y_{F B_{6}}$, respectively; $A_{i}$ is the magnitude of the $i^{\text {th }}$ spike in $F B_{6}$.

In order to compare the performance of these three new indicators with that of the three reported ones, i.e., RMSI and KURI (Wu et al., 2008) and RMSI3 (Tian et al., 2012), their values for 22 crack levels in $C L R=[1.29 \%, 58.06 \%]$ have been calculated. For each indicator, 22 values are obtained for all the considered crack levels, and they are formed as a value vector $V$. After that, $V$ is normalized with Eq. (8).

$$
V=\log _{10}\left(\frac{V}{\min (V)}\right)
$$

The normalized value vectors of the six indicators versus crack level are plotted in Figure 9. It's seen that the three developed indicators have higher increasing rates with regard to the crack level, which means they all outperform the three reported ones when tracking crack growth in CLR = $[1.29 \%, 58.06 \%]$. Especially, $S E_{F B_{m}}$ is most sensitive to reflect tooth crack growth and can be used to well assess crack severity level.

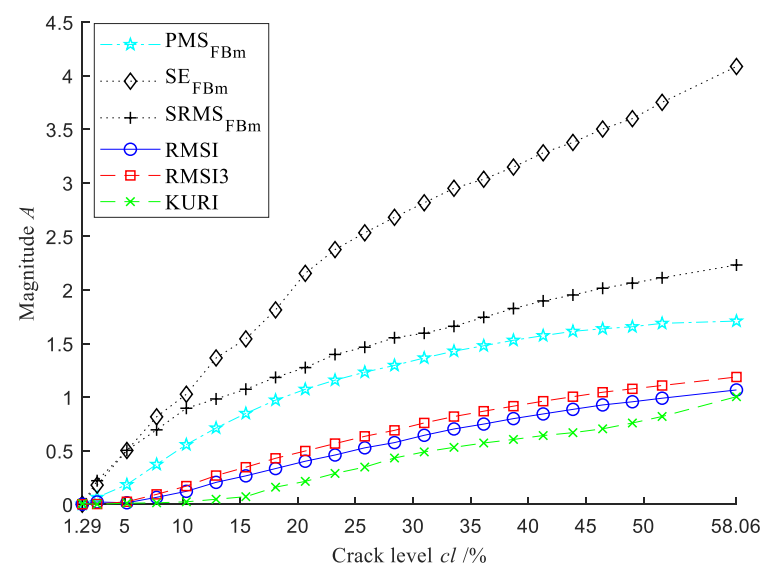

Figure 9. The normalized values of the six statistical indicators versus crack levels ( $C L R=[1.29 \%, 58.06 \%])$.

Besides, for early crack level in $C L R=[1.29 \%, 23.23 \%]$, the three proposed statistical indicators have much better performance than the three reported ones, which can be seen clearly in the zoomed-in plot shown in Figure 10. To further quantify these indicators' performance for early crack level assessment, their sensitivity to crack level is evaluated. The sensitivity is evaluated by calculating the increasing rate of the indicator magnitude with regard to the crack level. For the early crack level range $C L R=[1.29 \%, 23.23 \%]$, an sensitivity index (SI) is developed to evaluate the sensitivity of each indicator which is calculated with Equation (9). The higher the sensitivity index value is, the better performance the condition indicator has for early crack level assessment.

$$
S I=\frac{V_{\text {end }}-V_{\text {first }}}{c l_{\text {end }}-c l_{\text {first }}}
$$

where $V$ is the value vector of each condition indicator, $c l$ is the crack level in $C L R=[1.29 \%, 23.23 \%]$.

With Equation (9), SI values of the six indicators in Figure 10 are calculated and tabulated in Table 2. From Table 2, it's obvious that the $S I$ values of the three new condition indicators are much higher than the three reported ones, thus meaning they indeed have much better performance than the three reported ones. The result is consistent with the observation obtained from Figure 10.

Table 2. SI values of the six indicators.

\begin{tabular}{cccccc}
\hline$S E_{F B_{m}}$ & $S R M S_{F B_{m}}$ & $P M S_{F B_{m}}$ & RMSI & RMSI3 & KURI \\
\hline 10.83 & 6.37 & 5.28 & 2.09 & 2.58 & 1.31 \\
\hline
\end{tabular}

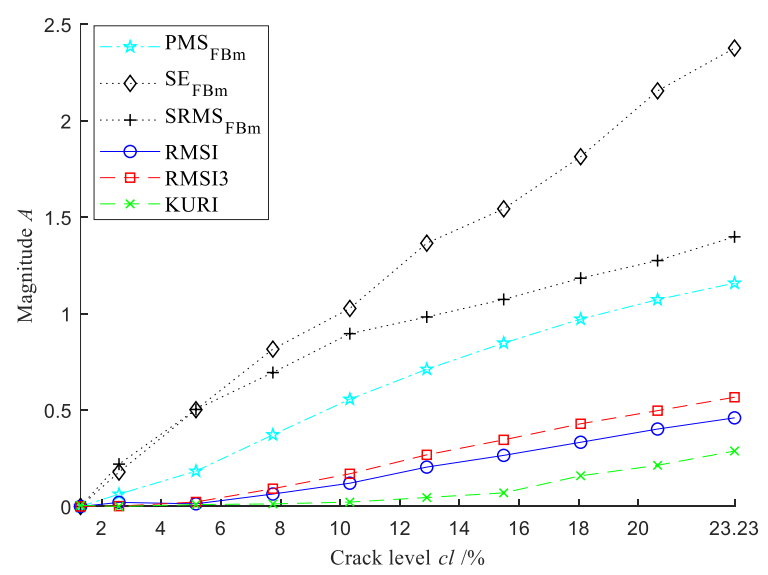

Figure 10. The normalized values of the six statistical indicators versus crack levels $(C L R=[1.29 \%, 23.23 \%])$.

\section{Conclusions}

This paper analyzes how crack level affects crack induced impulses. Three new effective statistical indicators are developed for crack severity level assessment in the early stage of crack propagation. The following conclusions are drawn. 
(1) In $C L R=[1.29 \%, 80 \%], y_{F B_{5}}$ and $y_{F B_{6}}$ are two dominant segments of crack induced impulse;

(2) In $C L R=[1.29 \%, 58.06 \%], y_{F B_{6}}$ is the most dominant segment. In $C L R=[1.29 \%, 23.23 \%]$, i.e., the early stage of crack propagation, $y_{F B_{6}}$ is most affected by crack level;

(3) In $C L R=[58.06 \%, 64.52 \%]$, impulse energy is gradually transferring from $y_{F B_{6}}$ to $y_{F B_{5}}$, which means this crack level range is a transition stage. The crack level in $[1.29 \%, 58.06 \%]$ is relatively small and the gear is relatively healthy, but when the crack level is in $[64.52 \%$, $80 \%$ ], the gear will become severely deteriorated. Therefore, in real industrial applications, this observation could be used to check which health state a gearbox is in, namely relatively healthy or severely deteriorated;

(4) In $C L R=[64.52 \%, 80 \%], y_{F B_{5}}$ is the most dominant segment;

(5) The three new indicators, namely $S E_{F B_{m}}, S R M S_{F B_{m}}$, and $P M S_{F B_{m}}$, have very good performance for tooth crack detection and severity assessment in the early stage of crack propagation, especially $S E_{F B_{m}}$.

The effectiveness of the three new indicators will be further checked with the experimental data or field data of gearboxes with localized tooth crack fault. If their effectiveness can still hold, then they could be used to conduct early tooth crack severity level assessment for gearboxes in the real industrial applications, thus improving the gearbox system reliability and reducing the operation and maintenance cost.

\section{ACKNOWLEDGEMENT}

Financial support from Future Energy Systems under Canada First Research Excellent Fund (FES-T11-P01) and China Scholarship Council is acknowledged. The authors would like to cordially thank the anonymous reviewers for their valuable comments and suggestions on this paper.

\section{REFERENCES}

Antoni, J., \& Randall, R. B. (2006). The spectral kurtosis: Application to the vibratory surveillance and diagnostics of rotating machines. Mechanical Systems and Signal Processing, 20(2), 308-331. https://doi.org/10.1016/j.ymssp.2004.09.002

Boyer, R., \& Abed-Meraim, K. (2004). Audio modeling based on delayed sinusoids. IEEE Transactions on Speech and Audio Processing, 12(2), 110-120. https://doi.org/10.1109/TSA.2003.819953

Hua, Y., \& Sarkar, T. K. (1990). Matrix pencil method for estimating parameters of exponentially damped/undamped sinusoids in noise. IEEE Transactions on Acoustics, Speech, and Signal Processing, 38(5), 814-824. https://doi.org/10.1109/29.56027
Kumaresan, R., \& Tufts, D. (1982). Estimating the parameters of exponentially damped sinusoids and polezero modeling in noise. IEEE Transactions on Acoustics, Speech, and Signal Processing, 30(6), 833-840. https://doi.org/10.1109/TASSP.1982.1163974

Lemmerling, P., \& Van Huffel, S. (2001). Analysis of the structured total least squares problem for Hankel/Toeplitz matrices. Numerical Algorithms, 27(1), 89-114. https://doi.org/10.1023/A:1016775707686

Li, Y., Ding, K., He, G., \& Lin, H. (2016). Vibration mechanisms of spur gear pair in healthy and fault states. Mechanical Systems and Signal Processing, 81, 183201. https://doi.org/10.1016/j.ymssp.2016.03.014

Liu, G., Quintero, J., \& Venkatasubramanian, V. M. (2007). Oscillation monitoring system based on wide area synchrophasors in power systems. 2007 IREP Symposium - Bulk Power System Dynamics and Control - VII. Revitalizing Operational Reliability, 1-13. https://doi.org/10.1109/IREP.2007.4410548

Potts, D., \& Tasche, M. (2010). Parameter estimation for exponential sums by approximate Prony method. Signal Processing, 90(5), 1631-1642. https://doi.org/10.1016/j.sigpro.2009.11.012

Randall, R. B. (1982). A new method of modeling gear faults. Journal of Mechanical Design, 104(2), 259-267. https://doi.org/10.1115/1.3256334

Tian, Z., Zuo, M. J., \& Wu, S. (2012). Crack propagation assessment for spur gears using model-based analysis and simulation. Journal of Intelligent Manufacturing, 23(2), 239-253. https://doi.org/10.1007/s10845-0090357-8

Tufts, D., \& Kumaresan, R. (1982). Singular value decomposition and improved frequency estimation using linear prediction. IEEE Transactions on Acoustics, Speech, and Signal Processing, 30(4), 671-675. https://doi.org/10.1109/TASSP.1982.1163927

Wang, L., \& Shao, Y. (2017). Fault mode analysis and detection for gear tooth crack during its propagating process based on dynamic simulation method. Engineering Failure Analysis, 71, 166-178. https://doi.org/10.1016/j.engfailanal.2016.11.003

Wang, WENYI. (2001). Early detection of gear tooth cracking using the resonance demodulation technique. Mechanical Systems and Signal Processing, 15(5), 887903. https://doi.org/10.1006/mssp.2001.1416

Wang, Wenyi, \& Wong, A. K. (2002). Autoregressive modelbased gear fault diagnosis. Journal of Vibration and Acoustics, 124(2), https://doi.org/10.1115/1.1456905

Wu, S., Zuo, M. J., \& Parey, A. (2008). Simulation of spur gear dynamics and estimation of fault growth. Journal of Sound and Vibration, 317(3), 608-624. https://doi.org/10.1016/j.jsv.2008.03.038

Zhao, M., \& Jia, X. (2017). A novel strategy for signal denoising using reweighted SVD and its applications to weak fault feature enhancement of rotating machinery. 
Mechanical Systems and Signal Processing, 94, 129-

147. https://doi.org/10.1016/j.ymssp.2017.02.036

\section{BIOGRAPHIES}

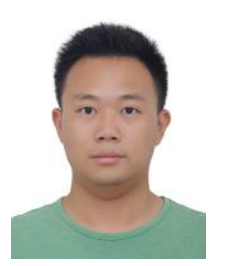

Xingkai Yang received the B.S. degree, Traffic Equipment and Information Engineering, Central South University, 2014; M.S. degree, Mechanical Engineering, Hunan University, 2017; both in Changsha, Hunan, China. He is currently working towards the Ph.D. degree at University of Alberta at Edmonton, Alberta, Canada. His research area includes advanced signal processing techniques, condition monitoring and fault diagnosis of mechanical systems.

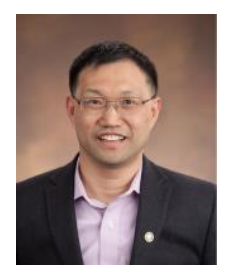

Ming J. Zuo received the Bachelor of Science degree in Agricultural Engineering in 1982 from Shandong Institute of Technology, China, and the Master of Science degree in 1986 and the Ph.D. degree in 1989 both in Industrial Engineering from Iowa State University, Ames, Iowa, U.S.A. He is currently Full Professor in the Department of Mechanical Engineering at the University of Alberta, Canada. His research interests include system reliability analysis, maintenance modeling and optimization, signal processing, and fault diagnosis. He is Department Editor of IISE Transactions, Regional Editor of International Journal of Strategic Engineering Asset Management, and Editorial Board Member of Reliability Engineering and System Safety, Journal of Traffic and Transportation Engineering, International Journal of Quality, Reliability and Safety Engineering, and International Journal of Performability Engineering. He is Fellow of the Institute of Industrial and Systems Engineers (IISE), Fellow of the Engineering Institute of Canada (EIC), Founding Fellow of the International Society of Engineering Asset Management (ISEAM), and Senior Member of IEEE.

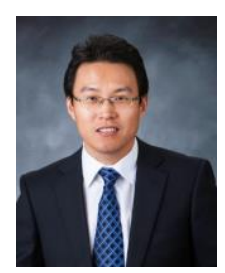

Zhigang Tian is currently an Associate Professor in the Department of Mechanical Engineering at the University of Alberta, Canada. He received his $\mathrm{PhD}$ degree from the University of Alberta in 2007. His research is focused on prognostics, pipeline integrity management, reliability, condition based maintenance, renewable energy systems, etc. 